\title{
Autoeficacia en la vida académica y rasgos
}

\author{
García-Méndez, Rosa María ${ }^{a *}$ y Rivera-Ledesma, Armandoa
}

\section{Artículo Original}

\section{Resumen}

\begin{abstract}
El objetivo del presente trabajo fue explorar la existencia de diferencias significativas en autoeficacia en la vida académica entre sujetos con rasgos psicopatológicos y sujetos que no presentan rasgos psicopatológicos en el contexto universitario mexicano, utilizando la Escala de Autoeficacia en la Vida Académica (EAVA) y el Inventario Multifásico de Personalidad de Minnesota (MMPI-2). La muestra estuvo compuesta por 251 estudiantes universitarios. Considerando los antecedentes, los sujetos con rasgos psicopatológicos obtuvieron puntuaciones más bajas en autoeficacia en la vida académica en comparación con sujetos que no presentan rasgos psicopatológicos. Destacaron las escalas asociadas a la integración social. En particular, Introversión social presentó puntuaciones más bajas en la mayoría de las sub-escalas EAVA, en especial: Liderazgo, Socialización y compañerismo, Logros profesionales, Creatividad e Innovación y Exposición frente a grupo. La investigación aportó evidencia sobre la importancia de la relación entre autoeficacia en la vida académica y rasgos psicopatológicos
\end{abstract}

Palabras clave: personalidad, autoeficacia académica adaptación universitaria, habilidades sociales.

Recibido el 14 de agosto de 2019. Aceptado el 29 de febrero de 2020

\begin{abstract}
Self-efficacy in academic life and psychopathological traits.

The aim of this study was to explore whether there are differences in self-efficacy in the academic life of students with psychopathological traits and students without these traits, based on results of the Self-Efficacy in Academic Life Scale (EAVA) and the Minnesota Multiphasic Personality Inventory (MMPI-2) instruments. We obtained and analyzed data from 251 Mexican university students. Individuals with psychopathological traits obtained significantly lower scores in the EAVA than students without psychopathological traits. Scales related to social integration were the most relevant. Among them, Social Introversion showed the lowest scores in most of the subscales of the EAVA, especially in the subscales: leadership, socialization and companionship, professional achievement, creativity and innovation, and exposure in front of a group. This study provides evidence that highlights the importance of the relation between psychopathological traits and the self-efficiency in academic life.
\end{abstract}

Keywords: personality, academic self-efficacy, adaptation to university, social skills.

Editaron este artículo: Jazmín Cevasco, Paula Abate, Sebastián Miranda y Sofia Sambre

\section{Introducción}

La autoeficacia académica se ha definido como la valoración que hace una persona con respecto a lo que puede o no hacer con sus habilidades y recursos personales para dominar las circunstancias cambiantes de su entorno académico (Bandura, 1995). En relación con esto, García-Méndez y Rivera-Ledesma (2020) conceptualizaron la autoeficacia en la vida académica siguiendo una concepción basada en factores cognitivos, conductuales y afectivos, emergentes en un contexto social donde las normas, valores, condicionantes económicos y materiales determinan exigencias adaptativas vinculadas directa $\mathrm{o}$ indirectamente con el quehacer académico del estudiante. Así, este constructo constituye una visión ampliada de la autoeficacia, que pone lo académico en relación con la constelación de factores circundantes que influyen sobre ella.

La autoeficacia en la vida académica puede ser concebida bajo una óptica multidimensional en la medida en que su definición remite a un

\footnotetext{
a Universidad Latina, Ciudad de México, México.

„Enviar correspondencia a: García-Méndez, R. M. E-mail: mailto:rmgarcia@unila.edu.mx

Citar este artículo como: García Méndez, R. M. y Rivera Ledesma, A. (2020). Autoeficacia en la vida académica y rasgos psicopatológicos. Revista Argentina de Ciencias del Comportamiento, 12(3), 41-58
} 
complejo sistémico de variables emergentes en un contexto sociocultural determinado. Dicho contexto impone exigencias adaptativas a múltiples estresores (Tosevsky, Milovancevic, \& Gajic, 2010), que el estudiante debe afrontar con base en sus creencias acerca de la eficacia de sus recursos - directa o indirectamente relacionados con lo académico - para obtener resultados satisfactorios. Estos procesos ocurren en diversos momentos adaptativos ligados al desarrollo humano, como la niñez (Menéndez, Jiménez, \& Lorence, 2008), la adolescencia (Silva, 2007) y la adultez (Uriarte, 2005), así como en relación con el nivel educativo del sujeto. Se trata de exigencias adaptativas particulares que confrontan al sujeto con nuevos retos de vida que han sido reconocidos por diferentes autores (Babío, 2011; Bereziartua, Zubiri, Intxausti, \& Odriozola, 2017; Borzone, 2017; Grøtan, Sund, \& Bjerkeset, 2019; Martínez, Torres, \& Huerta, 2005), y que proveen el escenario de lo que en este análisis se ha denominado "vida académica".

Considerando lo expuesto, puede proponerse que la vida académica está matizada por una adaptación enfocada a múltiples dimensiones (Chau \& Saravia, 2014; Sánchez, Álvarez, Flores, Arias, \& Saucedo, 2014), y mediada por las creencias acerca de la autoeficacia. Es decir, la adaptación a la vida académica no es un proceso segmentado, ya que el desempeño de un estudiante en su quehacer académico no está determinado exclusivamente por sus competencias escolares, sino por diversos procesos (Pajares \& Schunk, 2001).

Tomando en cuenta que el sujeto despliega estrategias adaptativas, funcionales o disfuncionales, que lo capacitan para el éxito o el fracaso (Moreno \& Chauta, 2012), puede considerarse que una de las variables mediadoras es la autoeficacia (Caprara \& Cervone, 2000).

La relación entre rasgos de personalidad y autoeficacia ha sido documentada por diferentes autores, si bien la tendencia general ha sido estudiar cada una de estas variables por separado. Investigaciones como las realizadas por Caprara, Vecchione, Alessandri, Gerbino y Barbaranelli (2011) y Fosse, Buch, Säfvenbom y Martinussen (2015) han destacado esto, así como el papel mediador de la autoeficacia en relación con los rasgos de personalidad, en especial la escrupulosidad [conscientiousness], y el rendimiento académico 0 laboral. Estos investigadores han sugerido que, si bien la autoeficacia y los rasgos de personalidad operan en diferentes niveles, las creencias acerca de la autoeficacia ejercen una función mediadora, permitiendo que los rasgos de personalidad se expresen como un comportamiento regulado al interactuar con el medio ambiente. En relación con esto, Fosse et al. (2015) proponen que las personas que creen en su propia capacidad transforman sus propias disposiciones básicas de carácter en comportamiento.

Para Caprara, Alessandri, Di Giunta, Panerai, y Eisenberg (2010), las creencias acerca de la autoeficacia median, al menos parcialmente, la influencia de los rasgos básicos en habilidades específicas y comportamientos, manteniendo los procesos cognitivos, afectivos y motivacionales que conducen al resultado deseado (i.e. un rendimiento exitoso). Para ellos, los rasgos básicos como la Escrupulosidad, la Apertura y las Creencias académicas de autoeficacia, pueden ser vistas como "capas de una arquitectura hipotética de la personalidad", en la que los rasgos básicos son disposiciones relativamente incondicionales y amplias que se refieren a lo que la persona 'tiene', y "la Autoeficacia académica es una estructura de conocimiento (es decir, un conjunto de creencias relacionadas con uno mismo) que opera en un nivel intermedio entre disposiciones amplias y comportamiento específico" (p.79).

Siguiendo a Tahmassian y Moghadam (2011), cada tipo o estructura de personalidad tiende a reaccionar al estrés del medio ambiente de forma diferencial con respuestas producto de la mediación de la autoeficacia. Las creencias acerca de la autoeficacia determinan los sentimientos, pensamientos, motivaciones y comportamientos de las personas. Es decir, un fuerte sentido de autoeficacia puede ayudar a combatir eficientemente el estrés y propiciar un sentimiento de bienestar. Se ha evidenciado (Gómez, Villegas de Posada, Barrera, \& Cruz, 2007) que las creencias de baja autoeficacia disponen a las personas a experiencias de fracaso, estrés mal manejado, depresión, y otras afecciones. En relación con esto, Tahmassian y Moghadam (2011) investigaron la relación entre Depresión y Autoeficacia utilizando distintos instrumentos para medir Autoeficacia social, Autoeficacia académica, Autoeficacia emocional, Autoeficacia física, así como Ansiedad rasgo- 
estado, Evitación social y Pensamientos negativos (preocupación). Los resultados indicaron una relación significativa y negativa entre el total de Autoeficacia, Autoeficacia física y Autoeficacia académica y Depresión, y una asociación similar entre el total de Autoeficacia, Autoeficacia física y Autoeficacia emocional y ansiedad. Se observó a su vez una relación negativa y significativa entre la Autoeficacia emocional y física y los Pensamientos negativos. Finalmente, la Autoeficacia social y física correlacionó negativa y significativamente con Evitación social.

Muris (2002) llegó a resultados similares en una muestra de adolescentes explorando la relación entre autoeficacia y rasgos de ansiedad y depresión. Sus hallazgos reportaron que bajos niveles de autoeficacia general fueron acompañados por altos niveles de rasgos de ansiedad / neuroticismo, síntomas de trastornos de ansiedad y síntomas de depresión. Adicionalmente, el investigador aportó evidencia acerca de que dominios específicos de autoeficacia están especialmente asociados con tipos particulares de problemas de ansiedad. Estos resultados sugieren que la autoeficacia social está fuertemente asociada a fobia social, la autoeficacia académica con la fobia escolar, y la autoeficacia emocional con el trastorno de ansiedad generalizada y el trastorno de pánico / somático. Por su parte, Karouei, Borjali, Jomehri y Sohrabi (2008) en una muestra de estudiantes de secundaria [High School], hallaron una relación inversa entre autoeficacia y el Sympton Check List (SCL90-R). Sus resultados indicaron que menores niveles de autoeficacia daban lugar a mayores niveles de distrés psicológico (somatización, obsesión-compulsión, sensibilidad interpersonal, depresión, ansiedad, hostilidad, fobias, pensamiento paranoide, y psicoticismo). Resultados similares han sido hallados con estudiantes universitarios. Burris, Brechting, Salsman y Carlson (2009) han señalado que estudiantes con altos niveles de distrés psicológico presentan alta ansiedad y baja autoeficacia.

De Miguel, Marrero, Fumero y Carballeira (2017) analizaron la existencia de diferencias en Inteligencia y Personalidad en estudiantes de licenciatura con alta y baja autoeficacia interpersonal. Sus resultados indicaron que las personas con baja autoeficacia interpersonal eran emocionalmente más inestables, más introvertidas, menos abiertas a la experiencia, menos cordiales, menos responsables en las tareas laborales / académicas, y puntuaban más alto en todos los trastornos de personalidad excepto el histriónico y el anancástico. En contraposición, las personas con autoeficacia interpersonal alta resultaron ser "más extrovertidas y responsables y con mayor puntuación en el rasgo límite y menor en el dependiente."

Grøtan et al. (2019) examinaron un grupo de estudiantes noruegos de ambos sexos menores de 35 años, a fin de evaluar si los alumnos que reportaron síntomas severos de problemas de salud mental, tenían un riesgo más alto de padecer una baja autoeficacia y un pobre progreso académico, en comparación con estudiantes que reportaron pocos síntomas o síntomas moderados. Los resultados mostraron una fuerte asociación entre síntomas severos de problemas de salud mental con una baja autoeficacia, y un riesgo Odds Ratio $(\mathrm{OR})=3.82$ veces mayor de llegar a presentarla (IC95\% 2.25 6.49).

Considerando estos antecedentes, puede proponerse que bajos niveles de autoeficacia podrían ser característicos de las alteraciones en estabilidad emocional y equilibrio psicológico en general, como las que es posible hallar en sujetos alcohólicos, adictos a alguna droga o que hubiesen sido expuestos por un periodo más o menos prolongado a situaciones límite con peligro para su vida. En un estudio que exploró la capacidad adaptativa en una muestra de pacientes alcohólicos y no alcohólicos, Bolzán, Kunzi y Regner (2000), compararon autoeficacia y rasgos de personalidad, a través del Inventario Multifásico de la Personalidad de Minnesota (MMPI-2; Bolzán et al., 2000). En cuanto a la percepción de autoeficacia, los investigadores hallaron que los sujetos alcohólicos puntuaron más bajo en defensividad, fuerza del Yo y dominancia. Con respecto a psicopatología asociada, los sujetos alcohólicos puntuaron más alto en hipocondriasis, depresión, desviación psicopática, paranoia, esquizofrenia, introversión social. Finalmente, hallaron puntuaciones significativamente más altas en alcoholismo, reconocimiento de adicción y adicción potencial. Los investigadores concluyeron que los sujetos alcohólicos se perciben menos autoeficaces, con mayor dificultad para enfrentar los problemas 
García Méndez, R. M. y Rivera Ledesma, A. / RACC, 2020, Vol. 12, º3, 41-58

cotidianos y con desconocimiento de sus propias capacidades, presentan problemas de autoafirmación, poca eficiencia en el manejo emocional de conflictos, y problemas de seguridad y confianza en sí mismos.

Chicharro, Pedrero y Pérez (2007) investigaron la autoeficacia presente en sujetos adictos en tratamiento en relación a sus rasgos de personalidad (Negativista, Autodestructivo, Esquizotípico y Límite). Los resultados indicaron asociaciones negativas, significativas e importantes entre ellas. Interesados en examinar la relación entre Estado de ánimo, Biotipo y Autoeficacia, Madueño et al. (2017) evaluaron a pacientes en alta de una unidad de rehabilitación cardiaca donde las personas cursan con situaciones extremas. Los resultados indicaron que los rasgos de personalidad correlacionaron de manera negativa y significativa con Autoeficacia en las variables Personalidad dependiente, Impulsivo inestable, Alteraciones de ánimo, Severidad de ansiedad, e Índice de depresión.

De acuerdo con las investigaciones citadas, parece existir amplia evidencia acerca de la relación entre rasgos psicopatológicos, personalidad y autoeficacia, y de la importancia de investigarla en contextos educativos en relación con el rendimiento académico y la adaptación del estudiante a múltiples estresores. Considerando estos antecedentes, el objetivo del presente trabajo fue explorar la relación entre autoeficacia en la vida académica y rasgos psicopatológicos de personalidad en el contexto universitario mexicano, y más específicamente, en sujetos de nuevo ingreso de la Ciudad de México y del Estado de Morelos. Esta investigación se ha enfocado en torno a saber si existen diferencias significativas en el grado de autoeficacia en la vida académica de sujetos con rasgos psicopatológicos y sujetos con rasgos de personalidad normales. Se espera que los sujetos con rasgos psicopatológicos presenten puntuaciones de autoeficacia en la vida académica significativamente menores que los sujetos sin estos rasgos.

\section{Método}

El diseño metodológico se estableció a partir de un modelo cuantitativo de tipo transversal, descriptivo correlacional - causal (Hernández, Fernández, \& Baptista, 2014; Kerlinger \& Lee, 2002), procediéndose como sigue:

\section{Participantes}

A partir de una población de 2979 sujetos de nuevo ingreso al nivel universitario a los cuales se les aplicó la EAVA y el MMPI-2 durante el periodo comprendido entre los años 2017 y principios de 2019, se estimó un tamaño muestral de 450 estudiantes con un margen de error del $4.2 \%$ y un nivel de confianza al $95.8 \%$. La muestra de carácter probabilístico se eligió siguiendo un procedimiento aleatorio simple operado mediante el programa IBM SPSS Statistics v. 22. Esta muestra inicial de 450 sujetos fue depurada considerando reelegir sólo aquellos protocolos cuyos resultados en el MMPI-2 estuviesen completamente resueltos y que fuesen válidos según los criterios establecidos para este instrumento (Sánchez, 2002). Ver Tabla 1.

Tabla 1.

Criterios de invalidación de protocolos del MMPI-2

\begin{tabular}{llc} 
Escala & Condición de invalidación & $\begin{array}{l}\text { Protocolos } \\
\text { Inválidos }\end{array}$ \\
\hline$?$ & $\begin{array}{l}\text { Número de preguntas sin } \\
\text { respuesta. }\end{array}$ & \\
& PD $\geq 30$ protocolo nulo. & 10
\end{tabular}

Escala L Uso de mentiras con respecto a la existencia de cualidades positivas en sí mismo. $T \geq 65$ indica un perfil inválido

$\begin{array}{lll}\text { Escala F } & \begin{array}{l}\text { Tendencia a la } \\ \text { exageración de los } \\ \text { problemas. }\end{array} \\ \mathrm{T} \geq 80 \text { indica un perfil } & 42 \\ \text { inválido } & \\ \text { Escala K } & \begin{array}{l}\text { Fingir buena imagen; } \\ \text { marcada defensividad. } \\ \mathrm{T}>70 \text { perfil invalidado }\end{array} \\ & \begin{array}{l}\text { Total de protocolos } \\ \text { inválidos }\end{array}\end{array}$

Nota. Basado en Meehl, 1946; Butcher y Williams, 1992; Avila y Jiménez, 1999; Graham, 1990, todos citados por Sánchez, 2002. T = Puntuación T. PD = Puntuación directa. $?=$ Preguntas sin respuesta

De los 450 casos se eliminaron 148 protocolos inválidos, más 51 incompletos. Es decir, en total, se descartaron 199 registros, quedando 251 registros válidos y completos. Se eligieron alumnos de nuevo ingreso en virtud de que se trata del segmento estudiantil más vulnerable en 
términos de adaptación universitaria y de mayor interés para la Universidad Latina - México en los procesos de acción tutorial. Por ello, la aplicación de la EAVA y el MMPI-2 está establecida para todos los alumnos en tal categoría. Las características de la muestra final se describen en la Tabla 2.

Tabla 2.

Características sociodemográficas de la muestra

\begin{tabular}{|c|c|c|}
\hline Edad & $M$ & $D E$ \\
\hline Femenino & 21.2 & 4.9 \\
\hline Masculino & 20.9 & 3.9 \\
\hline Total & 21.1 & 4.5 \\
\hline Sexo & $f$ & $\%$ \\
\hline Femenino & 140 & 55.8 \\
\hline Masculino & 111 & 44.2 \\
\hline Total & 251 & 100.0 \\
\hline \multicolumn{3}{|l|}{ Campus UL-M } \\
\hline Roma & 44 & 17.5 \\
\hline Sur & 133 & 52.6 \\
\hline Cuernavaca & 43 & 17.1 \\
\hline Cuautla & 31 & 12.4 \\
\hline Total & 251 & 100.0 \\
\hline \multicolumn{3}{|l|}{ Carrera } \\
\hline Administración & 41 & 16.3 \\
\hline Comunicación & 40 & 15.9 \\
\hline Contaduría & 22 & 8.8 \\
\hline Derecho & 59 & 23.5 \\
\hline Gastronomía & 18 & 7.2 \\
\hline Informática & 4 & 1.6 \\
\hline Mercadotecnia & 28 & 11.2 \\
\hline Pedagogía & 13 & 5.2 \\
\hline Psicología & 25 & 10.0 \\
\hline Turismo & 1 & .4 \\
\hline Total & 251 & 100.0 \\
\hline
\end{tabular}

Nota. $f=$ Frecuencia; $M=$ Media; $D E=$ Desviación estándar. UL-M = Universidad Latina - México.

\section{Instrumentos}

Además de una breve selección de preguntas de corte sociodemográfico se incluyeron sólo dos instrumentos de medición: La Escala de Autoeficacia en la Vida Académica (EAVA) (García-Méndez \& Rivera-Ledesma, 2020), y el Inventario Multifásico de Personalidad de Minnesota (MMPI-2, en su adaptación a México por Lucio, Reyes-Lagunes, \& Scott, 1994). Ampliamente utilizado y sujeto a estudios de validación en diferentes culturas y países del mundo, el MMPI-2 es uno de los instrumentos para la evaluación de la personalidad más robustos actualmente en uso. Este instrumento ha sido adaptado y evaluado psicométricamente en población mexicana en términos de validez, confiabilidad y estructura (Lucio et al., 1994), con adecuadas propiedades psicométricas. Para esta investigación se emplearon las escalas clínicas de Hipocondriasis, Depresión, Histeria, Desviaciones psicopáticas, Esquizofrenia, Manía, Paranoia, Psicastenia e Introversión social. La escala cinco de Intereses masculino femeninos no se empleó, en virtud de que no se trata de una categoría patológica sino de actitudes e intereses convencionales masculinos $\mathrm{o}$ femeninos (Zambrano-Guerrero, Caicedo-Padilla, \& Matabanchoy-Tulcán, 2015).

La Escala de Autoeficacia en la Vida Académica (EAVA; García-Méndez \& RiveraLedesma, 2020), ha sido validada en una muestra de 2926 estudiantes de nuevo ingreso captados durante el periodo de 2017 a 2019. Compuesta de 102 items distribuidos en 14 factores que explicaron el $59.5 \%$ de la varianza, empleando una escala tipo Likert de cuatro puntos (Valores de 1 a 4), obtuvo una confiabilidad Alfa entre .77 y .90 para sus factores, y de .89 para el total de la escala. La validez concurrente se estimó contra la Escala de Autoeficacia en Conductas Académicas (Blanco, Martínez, Zueck \& Gastélum 2011; Ornelas, Blanco, Peinado, \& Blanco, 2012), una escala desarrollada y validada en México contra la que obtuvo características robustas, reportó correlaciones estadísticamente significativas $(p<$ .01 ), entre $=.40$ y .60. Las escalas, número de ítems y puntuación máxima posible respectivamente, se describen a continuación: Logros profesionales, 12, 48; Liderazgo, 9, 36; Habilidades de investigación, 11, 44; Responsabilidad académica, 10, 40; Concentración y comprensión académicas, 9, 36; Creatividad e Innovación, 8, 32; Socialización y compañerismo, 10, 40; Exposición frente a grupo, 6, 24; Organización académica, 6, 24; Control de impulsos, 6, 24; Planeación académica, 5, 20; Aprendizaje fluido, 3, 12; Trabajo bajo presión, 3, 12; Asimilación de información, 4, 16.

\section{Procedimiento}

La investigación se basó en dos procesos distintos: por un lado, y como parte de la evaluación de estudiantes de nuevo ingreso a licenciatura, al momento de su ingreso se llevó a 
cabo la aplicación electrónica del MMPI-2 mediante un programa especialmente diseñado para ello. Alrededor de 3 meses después se aplicó la EAVA junto con datos sociodemográficos a los mismos estudiantes, a través del programa Formularios de Google. Las aplicaciones se llevaron a cabo en los centros de cómputo de cada campus de la institución anfitriona.

\section{Análisis de datos}

Los datos fueron analizados mediante los programas Excel e IBM SPSS Statistics v.22. EI análisis incluyó la estimación de la normalidad de las distribuciones de la EAVA y en el MMPI-2 y sus respectivas escalas. Si bien todas las escalas en el MMPI-2 mostraron una distribución normal, sólo el $50 \%$ de las escalas de la EAVA lograron satisfacer el criterio de normalidad mediante la prueba de Kolmogorov-Smirnoff. El resto se mostraron aproximadamente normales o fueron normalizadas. No se recurrió a estadística inferencial no paramétrica a fin de robustecer la estimación del tamaño del efecto.

Así, se realizaron contrastes mediante la prueba $\mathrm{t}$ para muestras independientes y la estimación del tamaño del efecto (Ledesma, Macbeth, \& Cortada de Kohan, 2008), siguiendo para su interpretación las sugerencias de Coe y Merino (2003), y para su estimación, la calculadora de Wilson (2001).

Para la clasificación de los sujetos de la muestra en normales y con rasgos psicopatológicos se siguieron los siguientes criterios: Álvarez (2016) ha señalado que el MMPI2 admite ser interpretado con base en sus diez primeras escalas clínicas y en acuerdo a sus puntuaciones T. Así, una puntuación igual o superior a T65 puede implicar desde problemas psicológicos significativos, hasta serios puntos frágiles de la personalidad. Puntuaciones menores o iguales a T64 y hasta T40 podrían identificar sujetos que no presentan rasgos psicopatológicos, y aquellas puntuaciones inferiores o iguales a T39 podrían no tener significado clínico en la mayoría de los casos. Para efectos de tamizaje y análisis estadístico se procedió a clasificar a los sujetos en cada variable de análisis en acuerdo con los criterios para sujetos que no presentan rasgos psicopatológicos y sujetos con síntomas psicopatológicos, sin considerar los casos restantes correspondientes a los perfiles hundidos.

\section{Aspectos éticos}

La aplicación de los instrumentos descritos fue precedida por la garantía de confidencialidad de los datos aportados por los alumnos, de la libertad de su participación y retiro anticipado de la encuesta en caso de así desearlo, habiéndose solicitado en todos los casos su consentimiento informado. En todo momento, se siguieron los lineamientos éticos correspondientes a los Principios éticos de los psicólogos y código de conducta de la American Psychological Association (2010), de la Sociedad Mexicana de Psicología en materia de investigación según se sigue del Código ético del psicólogo (Sociedad Mexicana de Psicología, 2010), en sus artículos sobre Confidencialidad (Artículos 132 al 137) y Consentimiento informado (Artículos 118 al 131), y en lo concerniente de la Declaración de Helsinki (World Medical Association, 2017) y el Código de Nüremberg (Universitas Miguel Hernández, 2019).

\section{Resultados}

Siguiendo los criterios de tamizaje ya descritos, se crearon dos grupos de sujetos. Por un lado, sujetos que no presentan rasgos psicopatológicos, y por otro, sujetos con rasgos psicopatológicos. A continuación, se estimó para cada uno de estos grupos la media y desviación estándar de cada una de las 14 escalas de autoeficacia en la vida académica, y se contrastaron mediante una prueba $t$ para grupos independientes, estimándose adicionalmente la magnitud del efecto con sus intervalos de confianza al 95\%. La Tabla 3 muestra los resultados obtenidos. 
García Méndez, R. M. y Rivera Ledesma, A. / RACC, 2020, Vol. 12, №3, 41-58

Tabla 3-A

Contraste de medias EAVA según sujetos con Rasgos Normales y Rasgos psicopatológicos en el MMPI-2

\begin{tabular}{|c|c|c|c|c|c|c|c|c|c|c|}
\hline \multirow{2}{*}{$\begin{array}{l}\text { Escalas } \\
\text { MMPI-2 }\end{array}$} & \multirow[b]{2}{*}{ Escalas EAVA } & \multicolumn{2}{|c|}{ RN } & \multicolumn{2}{|c|}{$\mathrm{RP}$} & \multirow[b]{2}{*}{$t$} & \multirow[b]{2}{*}{$g l$} & \multirow[b]{2}{*}{$p$} & \multirow[b]{2}{*}{$d$} & \multirow{2}{*}{$\begin{array}{c}\text { IC 95\% } \\
\text { LI, LS }\end{array}$} \\
\hline & & $M$ & $D E$ & $M$ & $D E$ & & & & & \\
\hline \multirow[t]{15}{*}{$\begin{array}{l}\text { Hipocondria } \\
\text { sis }\end{array}$} & Logros profesionales & 44.21 & 4.67 & 42.18 & 6.32 & 1.46 & 23 & .156 & .32 & {$[-.11, .76]$} \\
\hline & Liderazgo & 26.88 & 5.8 & 24.5 & 6.1 & 1.82 & 230 & .700 & .40 & {$[-.03, .84]$} \\
\hline & $\begin{array}{l}\text { Habilidades de } \\
\text { Investigación }\end{array}$ & 32.66 & 5.94 & 33.14 & 5.66 & .36 & 230 & .718 & .08 & {$[-.35, .51]$} \\
\hline & Responsabilidad & 36.4 & 4.58 & 36.09 & 4.74 & .30 & 230 & .764 & .06 & {$[-.37, .50]$} \\
\hline & $\begin{array}{l}\text { Concentración } \\
\text { Comprensión }\end{array}$ & 27.86 & 4.97 & 26.55 & 4.3 & 1.19 & 230 & .233 & .26 & {$[-.17, .70]$} \\
\hline & $\begin{array}{l}\text { Creatividad } \\
\text { Innovación }\end{array}$ & 26.9 & 4.5 & 24.4 & 6.00 & 2.35 & 230 & $.019^{*}$ & .52 & {$[.09, .97]$} \\
\hline & $\begin{array}{l}\text { Sociabilidad } \\
\text { Compañerismo }\end{array}$ & 33.27 & 5.2 & 31.9 & 5.93 & 1.15 & 230 & .250 & .25 & {$[-.18, .69]$} \\
\hline & $\begin{array}{l}\text { Exponer Frente a } \\
\text { Grupo }\end{array}$ & 17.78 & 4.2 & 16.82 & 3.68 & 1.07 & 230 & .283 & .24 & {$[-.20, .67]$} \\
\hline & Organización & 19.86 & 3.32 & 19.77 & 4.04 & .11 & 230 & .908 & .02 & {$[-.41, .46]$} \\
\hline & Control de impulsos & 19.75 & 3.03 & 19.77 & 3.02 & .03 & 230 & .976 & .00 & {$[-.43, .44]$} \\
\hline & Planeación & 15.99 & 2.91 & 15.32 & 3.64 & .99 & 230 & .321 & .22 & {$[-.21, .66]$} \\
\hline & Aprendizaje fluido & 8.93 & 1.84 & 8.91 & 1.68 & .04 & 230 & .962 & .00 & {$[-.43, .44]$} \\
\hline & Trabajo Bajo Presión & 9.68 & 2.11 & 8.73 & 2.33 & 1.99 & 230 & $.048^{*}$ & .45 & {$[.00, .89]$} \\
\hline & Asimilar información & 13.7 & 2.15 & 13.77 & 2.18 & .14 & 230 & .888 & .03 & {$[-.40, .47]$} \\
\hline & Total EAVA & 333.89 & 40.21 & 321.91 & 44.24 & 1.31 & 230 & .189 & .29 & {$[-.14, .73]$} \\
\hline \multirow[t]{15}{*}{ Depresión } & Logros profesionales & 43.89 & 4.86 & 42.05 & 6.56 & 1.19 & 19.95 & .247 & .28 & {$[-.18, .75]$} \\
\hline & Liderazgo & 26.78 & 5.66 & 21.74 & 7.08 & 3.62 & 214 & $.001^{\star \star}$ & .87 & {$[.39,1.35]$} \\
\hline & $\begin{array}{l}\text { Habilidades de } \\
\text { Investigación }\end{array}$ & 32.56 & 5.91 & 30.37 & 5.45 & 1.55 & 214 & .123 & .37 & {$[-.09, .84]$} \\
\hline & Responsabilidad & 35.86 & 4.77 & 37.26 & 4.29 & 1.23 & 214 & .220 & .29 & {$[-.17, .76]$} \\
\hline & $\begin{array}{l}\text { Concentración } \\
\text { Comprensión }\end{array}$ & 27.53 & 4.95 & 25.53 & 5.58 & 1.66 & 214 & .098 & .39 & {$[-.07, .87]$} \\
\hline & $\begin{array}{l}\text { Creatividad } \\
\text { Innovación }\end{array}$ & 26.6 & 4.62 & 24.47 & 5.86 & 1.87 & 214 & .063 & .44 & {$[-.02, .92]$} \\
\hline & $\begin{array}{l}\text { Sociabilidad } \\
\text { Compañerismo }\end{array}$ & 33.17 & 5.18 & 30.58 & 5.62 & 2.06 & 214 & $.041^{*}$ & .49 & {$[.02, .97]$} \\
\hline & $\begin{array}{l}\text { Exponer Frente a } \\
\text { Grupo }\end{array}$ & 17.81 & 3.88 & 14.47 & 3.39 & 3.61 & 214 & $.000^{*}$ & .87 & {$[.39,1.35]$} \\
\hline & Organización & 19.84 & 3.36 & 17.95 & 4.52 & 2.26 & 214 & $.024^{*}$ & .54 & {$[.07,1.02]$} \\
\hline & Control de impulsos & 19.6 & 3.12 & 20.16 & 2.41 & .75 & 214 & .453 & .18 & {$[-.29, .65]$} \\
\hline & Planeación & 15.85 & 2.94 & 14.47 & 3.33 & 1.91 & 214 & .057 & .45 & {$[-.01, .93]$} \\
\hline & Aprendizaje fluido & 8.98 & 1.73 & 7.68 & 2.16 & 3.06 & 214 & $.002^{* \star}$ & .73 & {$[.26,1.21]$} \\
\hline & Trabajo Bajo Presión & 9.55 & 2.1 & 9.11 & 2.49 & .86 & 214 & .390 & .30 & {$[-.26, .67]$} \\
\hline & Asimilar información & 13.63 & 2.1 & 12.95 & 2.93 & 1.29 & 214 & .195 & .30 & {$[-.16, .78]$} \\
\hline & Total EAVA & 331.66 & 40.09 & 308.79 & 45.15 & 2.34 & 214 & $.021^{*}$ & .56 & {$[.09,1.04]$} \\
\hline
\end{tabular}


García Méndez, R. M. y Rivera Ledesma, A. / RACC, 2020, Vol. 12, №3, 41-58

\begin{tabular}{|c|c|c|c|c|c|c|c|c|c|c|}
\hline \multirow[t]{15}{*}{ Histeria } & Logros profesionales & 43.7 & 5.15 & 44.5 & 4.75 & .42 & 188 & .668 & .15 & {$[-.55, .85]$} \\
\hline & Liderazgo & 26.52 & 5.86 & 30.38 & 4.98 & 1.82 & 188 & .069 & .65 & {$[-.05,1.36]$} \\
\hline & $\begin{array}{l}\text { Habilidades de } \\
\text { Investigación }\end{array}$ & 32.16 & 5.92 & 39.5 & 5.18 & 3.44 & 188 & $.001^{* *}$ & 1.24 & {$[.53,1.96]$} \\
\hline & Responsabilidad & 36.05 & 4.89 & 36.38 & 3.77 & .18 & 188 & .855 & .06 & {$[-.64, .77]$} \\
\hline & $\begin{array}{l}\text { Concentración } \\
\text { Comprensión }\end{array}$ & 27.32 & 4.98 & 31.25 & 3.45 & 2.20 & 188 & $.028^{*}$ & .79 & {$[.09,1.51]$} \\
\hline & $\begin{array}{l}\text { Creatividad } \\
\text { Innovación }\end{array}$ & 26.32 & 4.72 & 29.5 & 5.18 & 1.85 & 188 & .065 & .66 & {$[-.04,1.37]$} \\
\hline & $\begin{array}{l}\text { Sociabilidad } \\
\text { Compañerismo }\end{array}$ & 32.93 & 5.33 & 38.5 & 1.60 & 2.94 & 188 & $.004^{*}$ & 1.06 & {$[.34,1.77]$} \\
\hline & $\begin{array}{l}\text { Exponer Frente a } \\
\text { Grupo }\end{array}$ & 17.51 & 3.95 & 20.25 & 4.13 & 1.91 & 188 & $.050^{*}$ & .69 & {$[-.02,1.40]$} \\
\hline & Organización & 19.61 & 3.53 & 22.00 & 3.5 & 1.87 & 188 & .063 & .67 & {$[-.03,1.38]$} \\
\hline & Control de impulsos & 19.54 & 3.14 & 21.5 & 2.61 & 1.73 & 188 & .084 & .62 & {$[-.08,1.33]$} \\
\hline & Planeación & 15.74 & 3.1 & 17.75 & 2.86 & 1.79 & 188 & .074 & .64 & {$[-.06,1.35]$} \\
\hline & Aprendizaje fluido & 8.84 & 1.89 & 10.25 & 1.49 & 2.08 & 188 & $.038^{*}$ & .75 & {$[.04,1.47]$} \\
\hline & Trabajo Bajo Presión & 9.53 & 2.15 & 9.63 & 2.87 & .12 & 188 & .902 & .04 & {$[-.66, .75]$} \\
\hline & Asimilar información & 13.52 & 2.24 & 14.75 & 1.75 & 1.53 & 188 & .127 & .55 & {$[-.16,1.26]$} \\
\hline & Total EAVA & 329.29 & 41.54 & 366.13 & 41.27 & 2.45 & 188 & $.015^{*}$ & .88 & {$[.17,1.60]$} \\
\hline
\end{tabular}

Nota. RN = Rasgos normales; RP = Rasgos psicopatológicos; $M=$ Media; $D E=$ Desviación estándar; $t=$ Prueba $t$ para muestras independientes; * $p \leq .05 ;{ }^{* *} p \leq .01 ;{ }^{* * *} p \leq .001 ; \mathrm{gl}=$ Grados de libertad; $d=d$ de Cohen. IC $95 \%$

$=$ Intervalo de confianza al 95\%. LI = Límite inferior; LS = Límite superior.

Tabla 3-B.

Contraste de medias EAVA según sujetos con Rasgos Normales y Rasgos psicopatológicos en el MMPI-2

\begin{tabular}{|c|c|c|c|c|c|c|c|c|c|c|}
\hline \multirow{2}{*}{$\begin{array}{l}\text { Escalas } \\
\text { MMPI-2 }\end{array}$} & \multirow[b]{2}{*}{ Escalas EAVA } & \multicolumn{2}{|c|}{ RN } & \multicolumn{2}{|c|}{$\mathrm{RP}$} & \multirow[b]{2}{*}{$t$} & \multirow[b]{2}{*}{$g l$} & \multirow[b]{2}{*}{$p$} & \multirow[b]{2}{*}{$d$} & \multirow{2}{*}{$\begin{array}{l}\text { IC 95\% } \\
\text { LI, LS }\end{array}$} \\
\hline & & $M$ & $D E$ & $M$ & $D E$ & & & & & \\
\hline \multirow{12}{*}{$\begin{array}{c}\text { Desviacion } \\
\text { es } \\
\text { psicopática } \\
\text { s }\end{array}$} & $\begin{array}{l}\text { Logros } \\
\text { profesionales }\end{array}$ & 44.21 & 4.69 & 39.95 & 6.07 & 2.97 & 20 & $.007^{* *}$ & .71 & {$[.24,1.19]$} \\
\hline & Liderazgo & 27.19 & 5.83 & 24.84 & 5.69 & 1.67 & 220 & .095 & .40 & {$[-.07, .87]$} \\
\hline & $\begin{array}{l}\text { Habilidades de } \\
\text { Investigación }\end{array}$ & 33.06 & 5.86 & 30.21 & 7.25 & 1.98 & 220 & $.048^{*}$ & .47 & {$[.00, .95]$} \\
\hline & Responsabilidad & 36.34 & 4.43 & 33.16 & 6.40 & 2.12 & 19 & $.047^{*}$ & .51 & {$[.04, .98]$} \\
\hline & $\begin{array}{l}\text { Concentración } \\
\text { Comprensión }\end{array}$ & 28.03 & 4.89 & 25.00 & 5.47 & 2.55 & 220 & $.011^{* *}$ & .61 & {$[.14,1.09]$} \\
\hline & $\begin{array}{l}\text { Creatividad } \\
\text { Innovación }\end{array}$ & 26.81 & 4.64 & 25.47 & 5.42 & 1.18 & 220 & .237 & .28 & {$[-.18, .75]$} \\
\hline & $\begin{array}{l}\text { Sociabilidad } \\
\text { Compañerismo }\end{array}$ & 33.25 & 5.36 & 33.11 & 4.98 & .11 & 220 & .912 & .02 & {$[-.44, .49]$} \\
\hline & $\begin{array}{l}\text { Exponer Frente a } \\
\text { Grupo }\end{array}$ & 17.96 & 4.00 & 17.11 & 3.79 & .89 & 220 & .372 & .21 & {$[-.25, .68]$} \\
\hline & Organización & 20.07 & 3.33 & 17.05 & 4.25 & 3.69 & 220 & $.000^{* * *}$ & .88 & {$[.41,1.36]$} \\
\hline & $\begin{array}{l}\text { Control de } \\
\text { impulsos }\end{array}$ & 19.86 & 2.99 & 18.68 & 3.38 & 1.62 & 220 & .106 & .38 & {$[-.08, .86]$} \\
\hline & Planeación & 16.07 & 2.95 & 14.53 & 3.44 & 2.14 & 220 & $.033^{*}$ & .51 & {$[.04, .99]$} \\
\hline & Aprendizaje fluido & 9.05 & 1.79 & 8.68 & 2.13 & .84 & 220 & .400 & .20 & {$[-.26, .67]$} \\
\hline
\end{tabular}


García Méndez, R. M. y Rivera Ledesma, A. / RACC, 2020, Vol. 12, №3, 41-58

\begin{tabular}{|c|c|c|c|c|c|c|c|c|c|c|}
\hline & $\begin{array}{l}\text { Trabajo Bajo } \\
\text { Presión }\end{array}$ & 9.65 & 2.09 & 8.84 & 2.19 & 1.59 & 220 & .113 & .38 & {$[-.09, .85]$} \\
\hline & $\begin{array}{l}\text { Asimilar } \\
\text { información }\end{array}$ & 13.75 & 2.16 & 13.11 & 2.3 & 1.23 & 220 & .218 & .29 & {$[-.17, .76]$} \\
\hline & Total EAVA & 335.31 & 40.10 & 309.74 & 47.78 & 2.61 & 220 & $.010^{* *}$ & .62 & {$[.15,1.1]$.} \\
\hline \multirow[t]{15}{*}{ Paranoia } & $\begin{array}{l}\text { Logros } \\
\text { profesionales }\end{array}$ & 44.05 & 4.93 & 42.73 & 5.29 & 1.40 & 219 & .162 & .33 & {$[-.13, .80]$} \\
\hline & Liderazgo & 26.85 & 5.97 & 25.42 & 4.73 & 1.29 & 219 & .196 & .30 & {$[-.16, .78]$} \\
\hline & $\begin{array}{l}\text { Habilidades de } \\
\text { Investigación }\end{array}$ & 32.73 & 6.09 & 32.15 & 6.08 & .50 & 219 & .616 & .12 & {$[-.35, .59]$} \\
\hline & Responsabilidad & 36.34 & 4.6 & 34.67 & 5.42 & 1.86 & 219 & .063 & .44 & {$[-.02, .91]$} \\
\hline & $\begin{array}{l}\text { Concentración } \\
\text { Comprensión }\end{array}$ & 27.54 & 4.98 & 27.82 & 4.87 & .29 & 219 & .765 & .06 & {$[-.40, .53]$} \\
\hline & $\begin{array}{l}\text { Creatividad } \\
\text { Innovación }\end{array}$ & 26.63 & 4.73 & 26.97 & 4.31 & .38 & 219 & .703 & .09 & {$[-.37, .56]$} \\
\hline & $\begin{array}{l}\text { Sociabilidad } \\
\text { Compañerismo }\end{array}$ & 33.27 & 5.38 & 32.3 & 4.34 & .97 & 219 & .329 & .23 & {$[-.23, .70]$} \\
\hline & $\begin{array}{l}\text { Exponer Frente a } \\
\text { Grupo }\end{array}$ & 17.89 & 4.06 & 17.03 & 3.76 & 1.13 & 219 & .256 & .27 & {$[-.19, .74]$} \\
\hline & Organización & 19.78 & 3.51 & 19.03 & 3.66 & 1.11 & 219 & .265 & .26 & {$[-.20, .73]$} \\
\hline & $\begin{array}{l}\text { Control de } \\
\text { impulsos }\end{array}$ & 19.79 & 3.11 & 19.52 & 2.86 & .47 & 219 & .634 & .11 & {$[-.35, .58]$} \\
\hline & Planeación & 15.94 & 3.07 & 15.67 & 2.9 & .46 & 219 & .641 & .11 & {$[-.36, .58]$} \\
\hline & Aprendizaje fluido & 8.85 & 1.83 & 9.06 & 1.78 & .60 & 219 & .544 & .14 & {$[-.32, .61]$} \\
\hline & $\begin{array}{l}\text { Trabajo Bajo } \\
\text { Presión }\end{array}$ & 9.58 & 2.16 & 9.85 & 1.95 & .66 & 219 & .506 & .15 & {$[-.31, .62]$} \\
\hline & $\begin{array}{l}\text { Asimilar } \\
\text { información }\end{array}$ & 13.53 & 2.27 & 13.73 & 1.85 & .46 & 219 & .641 & .11 & {$[-.36, .58]$} \\
\hline & Total EAVA & 332.76 & 42.19 & 325.94 & 36.39 & .87 & 219 & .384 & .20 & {$[-.26, .67]$} \\
\hline \multirow[t]{13}{*}{$\begin{array}{l}\text { Psicasteni } \\
\text { a }\end{array}$} & $\begin{array}{l}\text { Logros } \\
\text { profesionales }\end{array}$ & 44.1 & 4.77 & 42.55 & 5.79 & 1.67 & 228 & .095 & .31 & {$[-.05, .68]$} \\
\hline & Liderazgo & 27.24 & 5.50 & 23.3 & 5.71 & 3.78 & 228 & $.000^{* * *}$ & .71 & {$[.34,1.09]$} \\
\hline & $\begin{array}{l}\text { Habilidades de } \\
\text { Investigación }\end{array}$ & 32.89 & 5.97 & 31.15 & 4.88 & 1.58 & 228 & .115 & .29 & {$[-.07, .66]$} \\
\hline & Responsabilidad & 36.19 & 4.44 & 35.85 & 4.67 & .40 & 228 & .683 & .07 & {$[-.29, .44]$} \\
\hline & $\begin{array}{l}\text { Concentración } \\
\text { Comprensión }\end{array}$ & 27.79 & 5.00 & 26.67 & 5.06 & 1.19 & 228 & .234 & .22 & {$[-.14, .59]$} \\
\hline & $\begin{array}{l}\text { Creatividad } \\
\text { Innovación }\end{array}$ & 26.93 & 4.58 & 25.64 & 5.21 & 1.47 & 228 & .142 & .27 & {$[-.09, .64]$} \\
\hline & $\begin{array}{l}\text { Sociabilidad } \\
\text { Compañerismo }\end{array}$ & 33.55 & 5.19 & 30.67 & 5.23 & 2.95 & 228 & $.003^{* *}$ & .55 & {$[.18, .93]$} \\
\hline & $\begin{array}{l}\text { Exponer Frente a } \\
\text { Grupo }\end{array}$ & 17.98 & 4.00 & 15.58 & 3.43 & 3.25 & 228 & $.001^{* * *}$ & .61 & {$[.24, .99]$} \\
\hline & Organización & 19.92 & 3.43 & 18.94 & 3.76 & 1.50 & 228 & .135 & .28 & {$[-.08, .65]$} \\
\hline & $\begin{array}{l}\text { Control de } \\
\text { impulsos }\end{array}$ & 19.93 & 3.04 & 18.91 & 2.93 & 1.80 & 228 & .073 & .33 & {$[-.03, .70]$} \\
\hline & Planeación & 16.02 & 2.96 & 15.03 & 2.62 & 1.79 & 228 & .074 & .33 & {$[-.03, .70]$} \\
\hline & Aprendizaje fluido & 9.01 & 1.78 & 8.39 & 1.78 & 1.82 & 228 & .070 & .34 & {$[-.02, .71]$} \\
\hline & $\begin{array}{l}\text { Trabajo Bajo } \\
\text { Presión }\end{array}$ & 9.61 & 2.14 & 9.24 & 2.00 & .93 & 228 & .353 & .17 & {$[-.19, .54]$} \\
\hline
\end{tabular}


García Méndez, R. M. y Rivera Ledesma, A. / RACC, 2020, Vol. 12, №3, 41-58

$\begin{array}{lccccccccc}\begin{array}{l}\text { Asimilar } \\ \text { información }\end{array} & 13.71 & 2.15 & 13.27 & 2.47 & 1.05 & 228 & .291 & .19 & {[-.17, .56]} \\ \text { Total EAVA } & 334.88 & 40.17 & 315.18 & 37.38 & 2.63 & 228 & .009^{* *} & .61 & {[.24, .99]}\end{array}$

Nota: RN = Rasgos normales; RP = Rasgos psicopatológicos; $M=$ Media; $D E=$ Desviación estándar; $t=$ Prueba $t$ para muestras independientes; * $p<=.05 ;{ }^{* *} p<=.01 ;{ }^{* * *} p<=.001 ; g l=$ Grados de libertad; $d=d$ de Cohen. IC $95 \%=$ Intervalo de confianza al 95\%. LI = Límite inferior; LS = Límite superior.

Tabla 3-C.

Contraste de medias EAVA según sujetos con Rasgos Normales y Rasgos psicopatológicos en el MMPI-2

\begin{tabular}{|c|c|c|c|c|c|c|c|c|c|c|}
\hline \multirow{2}{*}{$\begin{array}{l}\text { Escalas } \\
\text { MMPI-2 }\end{array}$} & \multirow[b]{2}{*}{ Escalas EAVA } & \multicolumn{2}{|c|}{ RN } & \multicolumn{2}{|c|}{$\mathrm{RP}$} & \multirow[b]{2}{*}{$t$} & \multirow[b]{2}{*}{$g l$} & \multirow[b]{2}{*}{$p$} & \multirow[b]{2}{*}{$d$} & \multirow{2}{*}{$\begin{array}{c}\text { IC 95\% } \\
\text { LI, LS }\end{array}$} \\
\hline & & $M$ & $D E$ & $M$ & $D E$ & & & & & \\
\hline \multirow[t]{15}{*}{ Esquizofrenia } & $\begin{array}{l}\text { Logros } \\
\text { profesionales }\end{array}$ & 44.27 & 4.72 & 42.00 & 5.74 & 2.23 & 44.07 & $.030^{*}$ & .40 & {$[.05, .76]$} \\
\hline & Liderazgo & 27.04 & 5.77 & 24.39 & 5.32 & 2.55 & 231 & $\begin{array}{c}.011^{*} \\
*\end{array}$ & .46 & {$[.11, .82]$} \\
\hline & $\begin{array}{l}\text { Habilidades de } \\
\text { Investigación }\end{array}$ & 32.61 & 5.91 & 31.75 & 5.18 & .82 & 231 & .413 & .14 & {$[-.20, .50]$} \\
\hline & Responsabilidad & 36.36 & 4.41 & 34.89 & 4.56 & 1.83 & 231 & .068 & .33 & {$[-.02, .68]$} \\
\hline & $\begin{array}{l}\text { Concentración } \\
\text { Comprensión }\end{array}$ & 27.78 & 5.00 & 26.56 & 4.98 & 1.35 & 231 & .178 & .24 & {$[-.11, .60]$} \\
\hline & $\begin{array}{l}\text { Creatividad } \\
\text { Innovación }\end{array}$ & 26.85 & 4.72 & 25.61 & 4.64 & 1.45 & 231 & .147 & .26 & {$[-.09, .61]$} \\
\hline & $\begin{array}{l}\text { Sociabilidad } \\
\text { Compañerismo }\end{array}$ & 33.28 & 5.34 & 31.89 & 4.82 & 1.45 & 231 & .147 & .26 & {$[-.09, .61]$} \\
\hline & $\begin{array}{l}\text { Exponer Frente } \\
\text { a Grupo }\end{array}$ & 17.9 & 4.03 & 15.86 & 3.35 & 2.86 & 231 & $.005^{*}$ & .52 & {$[.16, .88]$} \\
\hline & Organización & 20.05 & 3.41 & 18.5 & 3.75 & 2.46 & 231 & $\begin{array}{c}.014^{*} \\
*\end{array}$ & .45 & {$[.09, .80]$} \\
\hline & $\begin{array}{l}\text { Control de } \\
\text { impulsos }\end{array}$ & 20.00 & 2.30 & 18.31 & 2.81 & 3.14 & 231 & $\underset{*}{.002^{*}}$ & .57 & {$[.21, .93]$} \\
\hline & Planeación & 16.04 & 2.97 & 15.06 & 2.69 & 1.85 & 231 & .065 & .33 & {$[-.02, .69]$} \\
\hline & $\begin{array}{l}\text { Aprendizaje } \\
\text { fluido }\end{array}$ & 9.00 & 1.72 & 8.44 & 1.91 & 1.74 & 231 & .082 & .31 & {$[-.04, .67]$} \\
\hline & $\begin{array}{l}\text { Trabajo Bajo } \\
\text { Presión }\end{array}$ & 9.64 & 2.12 & 9.22 & 2.08 & 1.10 & 231 & .272 & .19 & {$[-.15, .55]$} \\
\hline & $\begin{array}{l}\text { Asimilar } \\
\text { información }\end{array}$ & 13.73 & 2.15 & 13.17 & 2.4 & 1.40 & 231 & .161 & .25 & {$[-.10, .60]$} \\
\hline & Total EAVA & 334.55 & 40.13 & 315.64 & 37.28 & 2.62 & 231 & $.009^{*}$ & .48 & {$[.12, .83]$} \\
\hline \multirow[t]{6}{*}{ Manía } & $\begin{array}{l}\text { Logros } \\
\text { profesionales }\end{array}$ & 43.91 & 4.55 & 44.48 & 5.35 & .82 & 237 & .409 & .11 & {$[-.16, .40]$} \\
\hline & Liderazgo & 26.88 & 5.87 & 27.67 & 5.12 & .97 & 237 & .332 & .13 & {$[-.14, .42]$} \\
\hline & $\begin{array}{l}\text { Habilidades de } \\
\text { Investigación }\end{array}$ & 32.98 & 5.93 & 33.03 & 5.85 & .06 & 237 & .950 & .00 & {$[-.27, .29]$} \\
\hline & Responsabilidad & 36.15 & 4.47 & 35.93 & 5.2 & .33 & 237 & .738 & .04 & {$[-.23, .32]$} \\
\hline & $\begin{array}{l}\text { Concentración } \\
\text { Comprensión }\end{array}$ & 27.74 & 4.92 & 28.18 & 4.86 & .61 & 237 & .539 & .08 & {$[-.19, .37]$} \\
\hline & $\begin{array}{l}\text { Creatividad } \\
\text { Innovación }\end{array}$ & 26.61 & 4.54 & 27.87 & 4.32 & 1.94 & 237 & .053 & .27 & {$[-.00, .56]$} \\
\hline
\end{tabular}


García Méndez, R. M. y Rivera Ledesma, A. / RACC, 2020, Vol. 12, №3, 41-58

\begin{tabular}{|c|c|c|c|c|c|c|c|c|c|c|}
\hline & \multirow{2}{*}{$\begin{array}{l}\text { Sociabilidad } \\
\text { Compañerismo } \\
\text { Exponer Frente } \\
\text { a Grupo }\end{array}$} & 33.26 & 5.03 & 33.87 & 5.53 & .80 & 237 & .419 & .11 & \multirow{2}{*}{$\begin{array}{l}{[-.16, .39]} \\
{[-.21, .35]}\end{array}$} \\
\hline & & 17.9 & 4.03 & 18.18 & 3.75 & .49 & 237 & .619 & .07 & \\
\hline & Organización & 19.99 & 3.38 & 20.03 & 3.38 & .08 & 237 & .932 & .01 & {$[-.27, .29]$} \\
\hline & $\begin{array}{l}\text { Control de } \\
\text { impulsos }\end{array}$ & 20.11 & 3.02 & 19.21 & 2.99 & 2.07 & 237 & $.039^{*}$ & .30 & {$[.02, .58]$} \\
\hline & Planeación & 15.98 & 2.91 & 16.19 & 3.00 & .51 & 237 & .608 & .07 & {$[-.20, .35]$} \\
\hline & $\begin{array}{l}\text { Aprendizaje } \\
\text { fluido }\end{array}$ & 9.03 & 1.73 & 9.06 & 1.88 & .12 & 237 & .905 & .01 & {$[-.26, .29]$} \\
\hline & $\begin{array}{l}\text { Trabajo Bajo } \\
\text { Presión }\end{array}$ & 9.52 & 2.17 & 10.03 & 1.89 & 1.69 & 237 & .091 & .24 & {$[-.03, .52]$} \\
\hline & $\begin{array}{l}\text { Asimilar } \\
\text { información }\end{array}$ & 13.7 & 2.15 & 13.76 & 2.14 & .18 & 237 & .852 & .02 & {$[-.25, .30]$} \\
\hline & Total EAVA & 333.75 & 39.29 & 337.48 & 41.38 & .64 & 237 & .517 & .09 & {$[-.19, .37]$} \\
\hline Introversión & $\begin{array}{l}\text { Logros } \\
\text { profesionales }\end{array}$ & 44.03 & 4.78 & 40.33 & 6.25 & 2.82 & 214 & $.005^{*}$ & .75 & {$[.23,1.29]$} \\
\hline social & Liderazgo & 26.73 & 5.75 & 20.8 & 5.32 & 3.81 & 214 & $.001^{* *}$ & 1.00 & {$[.50,1.57]$} \\
\hline & $\begin{array}{l}\text { Habilidades de } \\
\text { Investigación }\end{array}$ & 32.79 & 5.79 & 29.47 & 6.65 & 2.12 & 214 & $.035^{\star}$ & .57 & {$[.04,1.1]$.} \\
\hline & Responsabilidad & 36.05 & 4.82 & 35.87 & 4.79 & .14 & 214 & .887 & .03 & {$[-.48, .56]$} \\
\hline & $\begin{array}{l}\text { Concentración } \\
\text { Comprensión }\end{array}$ & 27.61 & 4.87 & 24.87 & 4.91 & 2.10 & 214 & $.037^{*}$ & .56 & {$[.04,1.09]$} \\
\hline & $\begin{array}{l}\text { Creatividad } \\
\text { Innovación }\end{array}$ & 26.72 & 4.52 & 22.4 & 6.07 & 2.70 & 15.17 & $\begin{array}{c}.016^{*} \\
*\end{array}$ & .72 & {$[.19,1.25]$} \\
\hline & $\begin{array}{l}\text { Sociabilidad } \\
\text { Compañerismo }\end{array}$ & 33.17 & 4.99 & 26.93 & 5.57 & 4.63 & 214 & $.000^{* *}$ & 1.20 & {$[.70,1.78]$} \\
\hline & $\begin{array}{l}\text { Exponer Frente } \\
\text { a Grupo }\end{array}$ & 17.55 & 3.94 & 14.8 & 3.32 & 2.63 & 214 & $.009^{*}$ & .71 & {$[.18,1.23]$} \\
\hline & Organización & 19.78 & 3.49 & 18.53 & 3.88 & 1.32 & 214 & .187 & .35 & {$[-.17, .87]$} \\
\hline & $\begin{array}{l}\text { Control de } \\
\text { impulsos }\end{array}$ & 19.75 & 3.02 & 19.00 & 2.33 & .94 & 214 & .348 & .25 & {$[-.27, .77]$} \\
\hline & Planeación & 15.98 & 2.95 & 14.2 & 2.86 & 2.25 & 214 & $.025^{\star}$ & .60 & {$[.07,1.13]$} \\
\hline & $\begin{array}{l}\text { Aprendizaje } \\
\text { fluido }\end{array}$ & 8.96 & 1.76 & 8.00 & 2.07 & 2.01 & 214 & $.046^{*}$ & .54 & {$[.01,1.07]$} \\
\hline & $\begin{array}{l}\text { Trabajo Bajo } \\
\text { Presión }\end{array}$ & 9.57 & 2.13 & 9.53 & 1.92 & .07 & 214 & .945 & .01 & {$[-.50, .54]$} \\
\hline & $\begin{array}{l}\text { Asımılar } \\
\text { información }\end{array}$ & 13.73 & 2.13 & 12.27 & 2.71 & 2.51 & 214 & $.013^{*}$ & .67 & {$[.15,1.20]$} \\
\hline & Total EAVA & 332.44 & 39.71 & 297.00 & 42.87 & 3.31 & 214 & .001 & .88 & {$[.36,1.42]$} \\
\hline
\end{tabular}

Nota. RN = Rasgos normales; RP = Rasgos psicopatológicos; $M=$ Media; $D E$ = Desviación estándar; $t=$ Prueba $t$ para muestras independientes; * $p<=.05 ;{ }^{* *} p<=.01 ;{ }^{* * *} p<=.001 ; g l=$ Grados de libertad; $d=d$ de Cohen. IC $95 \%$ = Intervalo de confianza al 95\%. LI = Límite inferior; LS = Límite superior.

Como es posible observar, las diferentes escalas clínicas, con excepción de Paranoia, presentan diferencias práctica y estadísticamente significativas entre las puntaciones de las distintas escalas de la EAVA de sujetos que no presentan rasgos psicopatológicos y las de aquellos con 
rasgos psicopatológicos. A excepción de la escala de Histeria, con puntuaciones más altas de autoeficacia coincidentes con puntuaciones más altas en la región de psicopatología, todas las demás presentan puntajes más elevados de autoeficacia en la vida académica en los sujetos que no presentan rasgos psicopatológicos (e.g., Total EAVA: 329.29 vs. 366.13 , respectivamente; $p=.015, d=.88$, respectivamente). En términos de la magnitud del efecto se evidencian diferencias que, en la mayoría de los casos, van de moderadas a importantes. La escala de Histeria obtuvo diferencias significativas con tamaños del efecto de moderados a importantes en Habilidades de investigación $(d=1.24)$, Concentración y comprensión $(d=0.79)$, Sociabilidad y compañerismo $(d=1.06)$, Exposición frente a grupo $(d=.68)$, Aprendizaje fluido $(d=.75)$ y Total EAVA $(d=.88)$. Cuando se analizan los resultados obtenidos en las comparaciones no significativas, se pudo observar que la única escala de personalidad que obtuvo tamaños del efecto moderados $(d>.50)$ fue la escala de Histeria (Liderazgo [ $d=.65]$, Creatividad e innovación [ $d=.66]$, Organización [ $d=.67]$, Control de impulsos [ $d=.62]$, Planeación [ $d=.64]$ y Asimilar información [ $d=.55]$ ).

En esta investigación, la escala de Introversión social del MMPI-2 es justamente la que presenta mayores diferencias significativas en la mayoría de los casos, tanto prácticas como estadísticas (e.g. Total EAVA: $p<.001, d=.88$ ), evidenciando importantes sentimientos de autoeficacia de orden social. En esta escala, las magnitudes de efecto más importantes se encuentran en Liderazgo ( $p=.001, d=1.04)$, Socialización y compañerismo $(p=.000, d=1.23)$, Logros profesionales $(p=.005, d=.75)$, Creatividad e Innovación ( $p=.016, d=.72)$ y Exposición frente a grupo $(p=.009, d=.71)$.

Es de destacar que las escalas de Liderazgo $(p=.000, d=.71)$, Socialización y Compañerismo $(p=.003, d=.55)$ y Exposición frente a grupo $(p=$ $.001, d=.61$ ), son también las únicas en las que se encuentran diferencias en la escala de Psicastenia, escala referente a la intensidad de la ansiedad, obsesiones, fobias e inhibición social e intelectual. Otra escala que suele presentar inhibición social y cognitiva, es la de Depresión, en la que aparecen las tres escalas de la EAVA comentadas arriba, más Organización y Aprendizaje fluido (con efectos entre $d=.54 \mathrm{y}$
.87).

La escala de Desviaciones psicopáticas es consistente con un nivel menor a lo normal en Logros profesionales $(p=.007, d=.71)$, Concentración $(p=.011, d=.61)$, Organización $(p$ $=.000, d=.88)$, Planeación $(p=.033, d=.51)$, Habilidades de investigación $(p=.048, \mathrm{~d}=.47)$ y Responsabilidad ( $p=.047, \mathrm{~d}=.51)$. Las escalas de Esquizofrenia y Manía conforme a lo teóricamente esperado, presentan menores niveles de autoeficacia en control de impulsos ( $d$ $=.57 \mathrm{y} \mathrm{d}=.30$, respectivamente), y la primera, en Organización $(p=.014, d=.45)$ y Logros profesionales ( $p=.031, \mathrm{~d}=.40)$; caracterizada por una tendencia a retraerse socialmente, presenta además menores niveles en Liderazgo $(p=.011, \mathrm{~d}$ $=.46)$ y Exposición frente a grupo $(p=.005, \mathrm{~d}=$ $.52)$.

\section{Discusión}

El objetivo del presente trabajo fue explorar la relación entre autoeficacia en la vida académica y rasgos psicopatológicos de personalidad en sujetos de nuevo ingreso a la Universidad de la Ciudad de México y del Estado de Morelos. Con este fin, se obtuvo un total de 251 participantes con protocolos completamente resueltos y válidos, conformados por el MMPI-2 y la EAVA. Considerando los resultados de estudios previos, se esperó que los sujetos con rasgos psicopatológicos presentasen puntuaciones de autoeficacia en la vida académica significativamente menores que los sujetos con puntuaciones normales.

Los resultados obtenidos sobre la relación entre Autoeficacia y Rasgos psicopatológicos de personalidad son consistentes con la literatura. Se obtuvieron diferencias estadística y prácticamente significativas entre las puntuaciones de la EAVA de sujetos que no presentan rasgos psicopatológicos y las puntuaciones de aquellos con rasgos psicopatológicos, evidenciándose que la autoeficacia en la vida académica reporta puntuaciones más bajas en estos últimos.

De Miguel et al. (2017) puntualizaron que sujetos con baja autoeficacia interpersonal eran también emocionalmente más inestables. A su vez, Tahmassian y Moghadam (2011), encontraron menores niveles de autoeficacia social, académica, emocional y física en sujetos con altos niveles de depresión y ansiedad. Por su parte, Karouei et al. (2008) y Burris et al. (2009) 
hallaron menores niveles de autoeficacia en presencia de mayores niveles de distrés psicológico. En muestras clínicas con personas sujetas a experiencias altamente estresantes y crónicas, o con importantes niveles de adicción a las drogas o alcohol, los resultados han sido similares (Bolzán et al., 2000; Chicharro et al., 2007; Madueño et al., 2017)

Los resultados de la presente investigación han aportado evidencia acerca de la relación entre 14 distintas áreas de autoeficacia en la vida académica, y nueve áreas de psicopatología, obteniendo hallazgos similares a los obtenidos por los investigadores citados. Adicionalmente, estos resultados también fueron consistentes con los reportados por Muris (2002), en cuanto a que dominios específicos de autoeficacia pueden estar asociados a rasgos de psicopatología específicos.

Los resultados de esta investigación son consistentes con la especificidad señalada por Muris (2002) en cuanto al número y tipo de áreas de autoeficacia en la vida académica identificadas para cada categoría de rasgos psicopatológicos. Por ejemplo, Introversión social sobresalió en importancia con diez áreas que presentaron menores niveles de autoeficacia, seguida de Desviaciones psicopáticas con seis, Esquizofenia y Depresión con cinco cada una, Histeria con cuatro y Psicastenia tres, Hipocondriasis dos, Manía una y Paranoia cero. En cada categoría de rasgos de psicopatología, la composición de las áreas involucradas fue distinta. Cualitativamente, los sentimientos referentes a bajos niveles de autoeficacia en áreas donde las habilidades sociales juegan un papel importante, como en la escala de Introversión social que hace referencia a "sentimientos de incomodidad en situaciones sociales, aislamiento, inadaptación general”, etc. (Butcher, Hass, Greene, \& Nelson, 2015; Graham, 2000), Esquizofrenia (Guízar, Saracco, \& Fresán, 2012), Psicastenia (Moral de la Rubia, 2014), y Depresión (Amezaga \& Saiz, 2015) fueron especialmente relevantes. A su vez, su impacto en Socialización y compañerismo, Liderazgo y Exposición frente a grupo fue importante, así como en Creatividad e Innovación y Logros profesionales.

La autopercepción del joven estudiante con respecto a su capacidad de Liderazgo, Socialización y compañerismo, y su habilidad para hacerse escuchar y ser aceptado durante la exposición académica frente a grupo, son fundamentales en los esfuerzos de adaptación sociocultural del alumno de nuevo ingreso a la universidad (Abello Riquelme et al., 2012; Holst, Galicia, Gómez, \& Degante, 2017), dado que contribuyen a su integración tanto al centro educativo como a grupos de pares (Madariaga \& Lozano, 2016), a la obtención de apoyo social (Zalazar-Jaime \& Cupani, 2016), y al progreso de sus sentimientos de aceptación e integración a la adultez joven.

Por otro lado, la escala de Histeria, a diferencia de las ocho escalas del MMPI-2 restantes, sólo incluyó cinco escalas con altos niveles de autoeficacia además de la escala Total EAVA, pero esta vez en el grupo de rasgos psicopatológicos en comparación con niveles más bajos en el grupo de sujetos que no presentan rasgos psicopatológicos, diferencias estadísticas significativas y con un tamaño del efecto importante. De Miguel et al. (2017) obtuvo hallazgos similares en su investigación bajo el término de Personalidad histriónica, equivalente en alguna medida al de Histeria en el MMPI-2. Sus resultados indicaron puntuaciones más altas en autoeficacia con respecto a sujetos con un perfil normal. La personalidad con rasgos histéricos suele ser histriónica, extrovertida, con amplias habilidades sociales y carisma personal, lo cual puede proveerle de sentimientos positivos de autoeficacia en una variedad de situaciones. Estos rasgos quedaron especialmente de manifiesto en las escalas de la EAVA que, aunque no resultaron ser estadísticamente significativas, si mostraron tamaños del efecto importantes. Esto puede explicar por qué es en esta escala donde los sujetos muestran puntajes más altos de autoeficacia en las puntuaciones $T$ más altas del MMPI-2.

Por otro lado, la escala de desviaciones psicopáticas dio cuenta de su impacto en varias escalas de la EAVA. Desviaciones psicopáticas evalúa:

Inadaptación social, ausencia de experiencias agradables, pobreza en los juicios de valores e ideas paranoides. Mide la inmadurez social, emocional y de responsabilidad y expresa la inconformidad por las normas que puede presentarse de forma encubierta o manifestarse con la rebelión (Zambrano-Guerrero et al., 2015, p. 248).

Este tipo de sintomatología debe ser tomada 
con cuidado. Se ha destacado que es fundamental mantener una actitud de cautela en la aplicación del constructo psicopático en la etapa juvenil, dado que algunos estudios refieren correlaciones débiles entre rasgos psicopáticos y reincidencia violenta (León-Mayer \& Zuñiga, 2012). Este tipo de rasgos pueden obedecer a problemas adaptativos, y su influencia en la autoeficacia en la vida académica parece ser importante, por lo que deben ser atendidos por la acción tutorial universitaria.

Por otro lado, es sabido que la depresión fomenta una visión negativa del futuro, del presente, y de uno mismo y las propias capacidades, por lo que la prevención y mejoramiento de cuadros depresivos leves (Mingote Adán, Gálvez Herrer, del Pino Cuadrado, \& Gutiérrez García, 2009) pueden impactar favorablemente la autoeficacia y el rendimiento escolar.

Se ha aportado evidencia acerca de la existencia de niveles más bajos de autoeficacia en la vida académica en sujetos con altos niveles de rasgos psicopatológicos (Burris et al., 2009; De Miguel et al., 2017; Grøtan et al., 2019; Karouei et al., 2008; Muris, 2002; Tahmassian \& Moghadam, 2011). Considerando estos antecedentes, los aspectos vinculados a las habilidades sociales han resultado especialmente importantes. Estos datos son orientadores para el diseño de estrategias de intervención formativa dentro del marco de la acción tutorial, dado que permiten tomar atención acerca del desarrollo de habilidades sociales (Huambachano \& Huaire, 2018), como un recurso fundamental para el fomento y facilitación de la integración de los estudiantes en grupos de pares, su permanencia en la institución mediante la estimulación de mejores percepciones en autoeficacia. Es decir, es importante considerar la necesidad de fortalecer las habilidades sociales, los sentimientos de aceptación e integración y los sentimientos de autoeficacia en la socialización, compañerismo, e intervención social (liderazgo, exposición académica), a fin de fortalecer la adaptación del estudiante universitario de nuevo ingreso.

Por último, es importante no perder de vista que las conductas poco apegadas a reglas, el consumo de sustancias, el bajo control de impulsos, etc., pueden ser síntomas asociados a la adolescencia tardía de algunos estudiantes que deben ser adecuadamente valorados y tratados (Vinet, 2010). Se ha sugerido (Tosevsky et al., 2010), que los estudiantes universitarios suelen estar sujetos a niveles de estrés capaces de producir resultados adversos en lo emocional, académico, y en la salud, y que es importante brindarles consejería tendiente a apoyarlos en la superación de sus problemas. Considerando los resultados de este estudio, es posible proponer que la psicopatología está vinculada con el quehacer académico de varias formas, y los programas de tutoría deben tomar nota de ello y ofrecer soluciones creativas, con el fin de brindar herramientas al estudiante para afrontar la vida académica.

Los antecedentes descritos y los resultados expuestos han dado cuenta de la importancia de la relación entre autoeficacia y personalidad en el contexto educativo, especialmente con respecto a rasgos psicopatológicos. Ambas variables contribuyen con información importante en diversos procesos de selección, evaluación y acción tutorial. En esas tareas, la Escala de Autoeficacia en la Vida Académica parece ser un instrumento útil.

Por otra parte, el empleo del MMPI-2 supone un proceso de aplicación de 567 preguntas que puede no resultar exitoso en algunos casos. En esta investigación, de 450 reportes analizados en la muestra aleatoria empleada, 148 (32.8\%), resultaron inválidos, probablemente por la influencia del contexto educativo de su aplicación. De esto se desprende que en las instituciones educativas el MMPI-2 quizá no debería ser un instrumento de evaluación de primer contacto. En su lugar, instrumentos como el SCL-90-R, instrumento cercano al MMPI-2, podría ser más conveniente tanto por su extensión, como por el tipo de información que proporciona; una medida de distrés psicológico puede ser más orientadora en la función tutorial. A partir de los resultados del SCL90-R puede requerirse o no un análisis más profundo mediante el empleo del MMPI-2, pero sólo en casos clínicamente especiales en que se presuma psicopatología significativa.

\section{Conclusiones}

$\mathrm{Ha}$ sido posible sumar evidencia a los resultados reportados en la literatura especializada en relación con la hipótesis planteada en esta investigación. Los resultados obtenidos permiten proponer que sujetos 
García Méndez, R. M. y Rivera Ledesma, A. / RACC, 2020, Vol. 12, №3, 41-58

mexicanos con altos puntajes en rasgos psicopatológicos poseen también menores puntajes en autoeficacia, en comparación con sujetos que no presentan rasgos psicopatológicos. Los hallazgos de esta investigación son relevantes para las instituciones educativas de nivel superior, porque llama la atención hacia la necesidad de considerar una importante disminución de la autoeficacia como indicativa de la posible existencia de problemas de orden emocional (e.g. depresión, ansiedad, mal manejo del estrés, etc.), que deben ser atendidos a fin de apoyar la capacidad adaptativa de los alumnos.

Cabe destacar, por otra parte, el comportamiento de la escalas de autoeficacia en la vida académica en relación con la escala de Histeria, donde la relación predicha se presentó en sentido inverso. Es decir, los sujetos con altos puntajes en rasgos psicopatólogicos histéricos, presentaron puntajes más altos de autoeficacia en la vida académica en comparación con sujetos que no presentan rasgos psicopatológicos. Este resultado nos lleva a considerar la posibilidad de que es precisamente la personalidad del sujeto la que impone sus rasgos esenciales a los sentimientos de autoeficacia. Esta no sería la primera ocasión en que la histeria hable para enseñarnos psicología.

En otro sentido, las escalas de autoeficacia en la vida académica relacionadas con situaciones de interacción social resultaron ser especialmente relevantes. Ante la presencia de rasgos psicopatológicos importantes, los sentimientos de autoeficacia en la vida académica en áreas de interacción social parecen verse vulnerados. En especial, Liderazgo, Exposición frente a grupo, Sociabilidad y compañerismo, juegan un papel importante en el desempeño profesional, y podrían ser objeto de programas tutoriales especializados en su desarrollo.

\section{Alcances y limitaciones}

El presente estudio se limitó a examinar la relación entre autoeficacia en la vida académica y rasgos psicopatológicos. Ha aportado evidencia acerca de la importancia de atender a aquellos estudiantes de nuevo ingreso con problemas emocionales, a fin de favorecer su adaptación a la vida universitaria.

La presente investigación no incluyó el constructo de ansiedad dentro de su diseño. Esta variable ha sido muy estudiada en el campo educativo (Cardona-Arias, Pérez-Restrepo, Rivera-Ocampo, Gómez-Martínez, \& Reyes, 2015; Castellanos, Guarnizo, \& Salamanca, 2011), especialmente en relación con la exposición oral ante grupos (Cordero \& Morales, 2016) y la realización de exámenes (Arco, López, Heilborn, \& Fernández, 2005), por lo que debería ser considerada en investigaciones futuras a fin de evaluar su importancia en los procesos adaptativos de los alumnos a la universidad, su vida profesional y sus sentimientos de autoeficacia en la vida académica.

Por otro lado, los resultados obtenidos describen, pero no explican, el efecto de los rasgos psicopatológicos sobre la autoeficacia y viceversa; tarea que habría de indagarse en futuros trabajos para comprender mejor la dinámica entre estos dos constructos. En futuras aproximaciones, sería conveniente estructurar muestras seleccionadas por tamaño y características idóneas para una mejor exploración del tema. La inclusión de variables como la ansiedad, el estrés producto de las presiones escolares y las estrategias de afrontamiento empleadas ante él en cada grupo de rasgos, podrían completar el análisis de la relación entre autoeficacia y rasgos psicopatológicos.

\section{Referencias}

Abello Riquelme, R., Díaz Mujica, A., Pérez Villalobos, M. V., Almeida, L. S., Lagos Herrera, I., González Puentes, J., \& Strickland, B. (2012). Vivencias e implicación académica en estudiantes universitarios: adaptación y validación de escalas para su evaluación. Estudios Pedagógicos, 38(2), 7-19. doi: $10.4067 /$ S0718-07052012000200001.

Álvarez, C. (2016). Guía para la Interpretación del MMPI-2. Buenos Aires, Argentina: Universidad de Buenos Aires.

American Psychological Association. (2010). 2010 Amendments to the 2002 "Ethical principles of psychologists and code of conduct". American Psychologist, 65(5), 493. doi: 10.1037/a0020168

Amezaga, P., \& Saiz, M. (2015). Tipos psicológicos y trastornos depresivos. Ciencias Psicológicas, 9(2), 141-152.

Arco, T. J. L., López, O. S., Heilborn, D. V. A., \& Fernández, M. F. D. (2005). Terapia breve en estudiantes universitarios con problemas de rendimiento académico y ansiedad: eficacia del modelo "La Cartuja". International Journal of Clinical and Health Psychology, 5(3), 589-608.

Babío, G. M. (2011). Orientación y transición entre 
etapas. En E. Martín Ortega \& I. Solé (Coords.), Orientación educativa: modelos y estrategias de intervención (pp. 151-167). España: Editorial Graó.

Bandura, A. (1995). Self-efficacy in changing societies. New York, US: Cambridge University Press. doi: 10.1017/CBO9780511527692

Bereziartua, J., Zubiri, A., Intxausti, N., \& Odriozola, A. (2017). Acción tutorial en la transición del alumnado de las escuelas del medio rural a la educación secundaria. Tendencias Pedagógicas, 29, 189-210. doi: 10.15366/tp2017.29.008

Blanco, V. H., Martínez, M. M., Zueck, E. M. C., \& Gastélum, C. G. (2011). Análisis psicométrico de la escala Autoeficacia en Conductas Académicas en estudiantes universitarios de primer ingreso. Revista Actualidades Investigativas en Educación, 11(3), 1-27. doi: 10.15517/aie.v11i3.10214

Bolzán, C., Kunzi, I., \& Regner, E. (2000). La evaluación de rasgos de personalidad y autoeficacia de una muestra argentina de pacientes alcohólicos. Interdisciplinaria, 17(2), 7193.

Borzone, V. M. A. (2017). Autoeficacia y vivencias académicas en estudiantes universitarios. Acta Colombiana de Psicología, 20(1), 266-274. doi: 10.14718/ACP.2017.20.1.13

Burris, J. L., Brechting, E., Salsman, J., \& Carlson, C. R. (2009). Factors associated with the psychological wellbeing and distress of university students. Journal of American College Health, 57(5), 536-544. doi: 10.3200/JACH.57.5.536-544

Butcher, J. N., Hass, G. A., Greene, R. L., \& Nelson, L. D. (2015). Using the MMPI-2 in forensic assessment. Washington, D.C.: American Psychological Association.

Caprara, G., Alessandri, G., Di Giunta, L., Panerai, L., \& Eisenberg, N. (2010). The contribution of agreeableness and self-efficacy beliefs to prosociality. European Journal of Personality, 24(1), 36-55. doi: 10.1002/per.739

Caprara, G., \& Cervone, D. (2000). Personality: Determinants, Dynamics, and Potentials. Cambridge, US: Cambridge University Press. doi: 10.1017/CBO9780511812767

Caprara, G., Vecchione, M., Alessandri, G., Gerbino, M., \& Barbaranelli, C. (2011). The contribution of personality traits and self-efficacy beliefs to academic achievement: A longitudinal study. British Journal of Educational Psychology, 81(1), 78-96. doi: 10.1348/2044-8279.002004

Cardona-Arias, J., Pérez-Restrepo, D., Rivera-Ocampo, S., Gómez-Martínez, J., \& Reyes, A. (2015). Prevalencia de ansiedad en estudiantes universitarios. Diversitas. Perspectivas en Psicología, 11(1), 79-89. doi: 10.15332/s17949998.2015.0001.05

Castellanos, C., Guarnizo, C., \& Salamanca, C. (2011).
Relación entre niveles de ansiedad y estrategias de afrontamiento en practicantes de psicología de una universidad colombiana. International Journal of Psychological Research, 4(1), 50-57.

Chau, C., \& Saravia, J. C. (2014). Adaptación universitaria y su relación con la salud percibida en una muestra de jóvenes de Perú. Revista Colombiana de Psicología, 23(2), 269-284. doi: 10.15446/rcp.v23n2.41106

Chicharro, J. R., Pedrero, E. J. P., \& Pérez, M. L. (2007). Autoeficacia para resistirse al consumo de sustancias como predictora de resultados de tratamiento y su relación con variables de personalidad: estudio de una muestra de adictos con el DTCQ, el VIP y el MCMI-II. Adicciones, 19(2), 141-151. doi: 10.20882/adicciones.312

Coe, R., \& Merino, S. C. (2003). Magnitud del Efecto: Una guía para investigadores y usuarios. Revista de Psicología de la PUCP, 21(1), 145-177.

Cordero, B. D., \& Morales, R. Y. (2016). Estrategias para reducir la ansiedad durante las actividades orales en estudiantes de inglés. Revista de Lenguas Modernas, 25, 253-274. doi: 10.15517/rlm.v0i25.27705

De Miguel, A., Marrero, R., Fumero, A., \& Carballeira, M. (2017). El Papel de la Personalidad y la Inteligencia en la Autoeficacia Interpersonal. Revista Iberoamericana de Diagnóstico y Evaluación, 44(2), 16-27. doi: 10.21865/RIDEP44.2.02

Fosse, T., Buch, R., Säfvenbom, R., \& Martinussen, M. (2015). The impact of personality and self-efficacy on academic and military performance: The mediating role of self-efficacy. Journal of Military Studies, 6(1), 1-19. doi: 10.1515/jms-2016-0197

García-Méndez, R. M., \& Rivera-Ledesma, A. (2020). Escala de Autoeficacia en la Vida Académica: Propiedades psicométricas en estudiantes de nuevo ingreso a pregrado. Zenodo. Publicación anticipada en línea. doi: 10.5281/zenodo.3661265

Gómez, V., Villegas de Posada, C., Barrera, F., \& Cruz, J. E. (2007). Factores predictores de bienestar subjetivo en una muestra colombiana. Revista Latinoamericana de Psicología, 39(2), 311-325.

Graham, J. (2000). MMPI-2, assessing personality and psychopathology (3ra. Ed.). Oxford, NY: Oxford University Press.

Grøtan, K., Sund, E., \& Bjerkeset, O. (2019). Mental health, academic self-efficacy and study progress among college students - The SHoT study, Norway. Frontiers in Psychology, 10, 45. doi: 10.3389/fpsyg.2019.00045

Guízar, S. D. P., Saracco, A. R., \& Fresán, O. A. (2012). Rasgos de personalidad en pacientes con esquizofrenia. Salud Mental, 35(4), 339-344.

Hernández, S. R., Fernández, C. C., \& Baptista, L. P. (2014). Metodología de Investigación (6 $6^{\mathrm{a}} \mathrm{Ed}$.). 
García Méndez, R. M. y Rivera Ledesma, A. / RACC, 2020, Vol. 12, №3, 41-58

México: McGraw Hill.

Holst, M. I. C., Galicia, B. Y., Gómez, V. G., \& Degante, G. A. (2017). Las habilidades sociales y sus diferencias en estudiantes universitarios. Vertientes, Revista Especializada en Ciencias de la Salud, 20(2), 22-29.

Huambachano, C. C. A. M., \& Huaire, I. E. J. (2018). Desarrollo de habilidades sociales en contextos universitarios. Horizonte de la Ciencia, 8(14), 123130.

doi:

10.26490/uncp.horizonteciencia.2018.14.430

Karouei, R., Borjali, A., Jomehri, F., \& Sohrabi, F. (2008). Relationship between general self-efficacy and psychopathological symptoms in high school students. Journal of Psychology, 12(2), 122-135.

Kerlinger, F. N., \& Lee, H. B. (2002). Investigación del comportamiento. Métodos de investigación en ciencias sociales ( $4^{\mathrm{a}} \mathrm{Ed}$.). México: McGrawHill.

Ledesma, R., Macbeth, G., \& Cortada de Kohan, N. (2008). Tamaño del efecto: Revisión teórica y aplicaciones con el sistema estadístico vista. Revista Latinoamericana de Psicología, 40(3), 425439.

León-Mayer, E., \& Zuñiga, D. P. (2012). Características psicopáticas en la adolescencia: sistematización teórica. Universitas Psychologica, 11(4), 11971207.

Lucio, E., Reyes-Lagunes, I., \& Scott, R. L. (1994). MMPI-2 for México: Translation and Adaptation. Journal of Personality Assessment, 63(1), 105-116. doi: 10.1207/s15327752jpa6301_9

Madariaga, C., \& Lozano, J. J. E. (2016). El apoyo social en estudiantes universitarios y su relación con las comunicaciones cara a cara y las comunicaciones mediadas por las tecnologías de la información y la comunicación (TIC). Psicogente, 19(35), 47-62. doi: 10.17081/psico.19.35.1208

Madueño, A. J .C., Mellado, M. L. F., Delgado, J. P., Muñoz, M. A., Pardos, M. L., \& Saez, L. G. (2017). Autoeficacia percibida, rasgos de personalidad y biotipos previos a programa de rehabilitación cardíaca en atención primaria de salud. Enfermería Clínica, 27(6), 335-400. doi: 10.1016/j.enfcli.2017.04.004

Martínez, L. J. F., Torres, P. M. A., \& Huerta, M. R. M. (2005). Los Planes de Acción Tutorial en la Universidad Autónoma de San Luis Potosí. La trayectoria académica como eje de construcción. Revista Mexicana de Orientación Educativa, 3(5), 34-37.

Menéndez, S., Jiménez, L., \& Lorence, B. (2008). Familia y adaptación escolar durante la infancia. Revista de Educación, 10, 97-110.

Mingote Adán, J. C., Gálvez Herrer, M., del Pino Cuadrado, P., \& Gutiérrez García, M. D. (2009). El paciente que padece un trastorno depresivo en el trabajo. Medicina y Seguridad en el Trabajo,
55(214), 41-63. doi: 10.4321/S0465$546 \times 2009000100004$

Moral de la Rubia, J. (2014). Dimensiones psicopatológicas de las escalas clínicas del MMPI2. Revista de Psicopatología y Psicología Clínica, 19(1), 45-62.

doi: 10.5944/rppc.vol.19.num.1.2014.12982

Moreno, M. J. H., \& Chauta, R. L. C. (2012). Funcionalidad familiar, conductas externalizadas y rendimiento académico en un grupo de adolescentes de la ciudad de Bogotá. Psychologia. Avances de la Disciplina, 6(1), 155-166.

Muris, P. (2002). Relationships between self-efficacy and symptoms of anxiety disorders and depression in a normal adolescent sample. Personality and Individual Differences, 32(2), 337-348. doi: 10.1016/S0191-8869(01)00027-7

Ornelas, C. M., Blanco, V. H., Peinado, P. J. E., \& Blanco, O. J. R. (2012). Autoeficacia percibida en conductas académicas en universitarios. Un estudio respecto de alumnos de educación física. Revista Mexicana de Investigación Educativa, 17(54), 779-791.

Pajares, F., \& Schunk, D. (2001). Self-beliefs and school success: Self-efficacy, self-concept, and school achievement. En R. J. Riding \& S. G. Rayner (Eds.), International perspectives on individual differences, Vol. 2 Self- perception (pp. 239-265). Westport, CT, US: Ablex Publishing.

Sánchez, C. (2002). Sensibilidad de las escalas e indicadores de validez en el perfil de personalidad del MMPI-2. Salamanca, España: Ediciones Universidad de Salamanca.

Sánchez, P. M. L., Álvarez, C. A., Flores, C. T., Arias, R. J., \& Saucedo, G. M. (2014). El reto del estudiante universitario ante su adaptación y autocuidado como estrategia para disminuir problemas crónicos degenerativos. Educación y Salud, 2(4). doi: 10.29057/icsa.v2i4.754

Silva, D. I. (2007). La adolescencia y su interrelación con el entorno. Madrid, España: Instituto de la Juventud.

Sociedad Mexicana de Psicología. (2010). Código Ético del Psicólogo. México: Trillas

Tahmassian, K., \& Moghadam, J. N. (2011). Relationship between self-efficacy and symptoms of anxiety, depression, worry and social avoidance in a normal sample of students. Iranian Journal of Psychiatry Behavioral Sciences, 5(2), 91- 98.

Tosevsky, D., Milovancevic, M., \& Gajic, S. D. (2010). Personality and psychopathology of university students. Current Opinion in Psychiatry, 23(1), 4852. doi: $10.1097 /$ yco.0b013e328333d625.

Universitas Miguel Hernández. (2019). Código de Nuremberg. Recuperado de: http://oep.umh.es/files/2013/12/C\%C3\%B3digo_N $\%$ C3\%BCremberg.pdf 
García Méndez, R. M. y Rivera Ledesma, A. / RACC, 2020, Vol. 12, №3, 41-58

Uriarte, A. (2005). En la transición a la edad adulta. Los adultos emergentes. International Journal of Developmental and Educational Psychology, 3(1), 145-160.

Vinet, E. (2010). Psicopatía infanto-juvenil: avances en conceptualización, evaluación e intervención. Terapia Psicológica, 28(1), 109-118. doi: 10.4067/S0718-48082010000100010

Wilson, D. B. (2001). Practical Meta-Analysis effect size calculator. Recuperado de: http://www.campbellcollaboration.org/escalc/html/E ffectSizeCalculator-ESTypes.php

World Medical Association (WMA). (2017). Declaración de Helsinki de la AMM - Principios éticos para las investigaciones médicas en seres humanos. Recuperado de: https://www.wma.net/es/policiespost/declaracion-de-helsinki-de-la-amm-principioseticos-para-las-investigaciones-medicas-en-sereshumanos/

Zalazar-Jaime, M. F., \& Cupani, M. (2016). Adaptación de dos medidas de apoyo social en estudiantes argentinos. Actualidades en Psicología, 30(120), 57-70. doi: 10.15517/ap.v30i120.21809

Zambrano-Guerrero, C. A., Caicedo-Padilla, D. A., \& Matabanchoy-Tulcán, S. A. (2015). Revisión sistemática sobre el Inventario Multifásico de Personalidad de Minnesota (MMPI). Universidad y Salud, 17(2), 246-261. doi: 10.22267/rus.151702.9 